DOI: https://doi.org/10.24297/jap.v16i1.8373

\title{
Random Stability of Quadratic Functional Equations
}

\author{
Mee Kwang Kang \\ Department of Mathematics, Dong-eui University, Busan 47340, Republic of Korea \\ mee@deu.ac.kr
}

\begin{abstract}
In this paper, we investigate the generalized Hyers-Ulam stability on random p-normed spaces associated with the following generalized quadratic functional equation $f(k x+y)+f(k x-y)=2 f(x+y)+2 f(x-y)+$ $2\left(k^{2}-2\right) f(x)-2 f(y)$, where $\mathrm{k}$ is a fixed positive integer via two methods
\end{abstract}

Keywords: Random P-Normed Space, Quadratic Functional Equation, Generalized Hyers-Ulam Stability, Direct Method, Fixed Point Method

\section{Introduction}

The theory of random normed spaces is important as a generalization of the deterministic results of normed spaces and also in the study of random operator equations. The notion of a random normed space corresponds to the situations when we do not know exactly the norm of the point and we know only probabilities of possible values of this norm. The random normed spaces may provide us the appropriate tools to study the geometry of nuclear physics and have useful applications in quantum particle physics.

In 1940, Ulam [1] suggested the stability problem of functional equations concerning the stability of group homomorphisms as follows: When is it true that a function that approximately satisfies a functional equation must be close to an exact solution of the equation? If the problem accepts a solution, we say the equation is stable. The famous Ulam stability problem was partially solved by Hyers [2] for the linear functional equation of Banach spaces. Hyers's theorem was generalized by Aoki [3] for additive mappings and by Rassias [4] for linear mappings by considering an unbounded Cauchy difference. A generalization of the Rassias theorem was obtained by Găvruta [5] by replacing the unbounded Cauchy difference by a general control function in the spirit of Rassias approach. The paper of Rassias has provided a lot of influence in the development of what we call generalized Hyers-Ulam stability of functional equations. Cădariu and Radu [6] applied the fixed-point method to investigation of the Jensen functional equation. They could present a short and simple proof (different from the direct method initiated by Hyers in 1941) for the generalized Hyers-Ulam stability of Jensen functional equation and for quadratic functional equation.

The quadratic function $\mathrm{f}(\mathrm{x})=\mathrm{c} x^{2}$ satisfies the functional equation

$f(x+y)+f(x-y)=2 f(x)+2 f(y)$

and therefore, the functional equation (1.1) is called the quadratic functional equation. The generalized HyersUlam-Rassias stability theorem for the quadratic functional equation (1.1) was proved by [7, 8, 9] and the references therein. The stability problems of several functional equations have been extensively investigated by a number of authorss, and there are many interesting results concerning this paper $[10,11,12,13]$. Before presenting our results, we introduce some basic facts concerning of random normed spaces as in [14, 15]. A function $\mathrm{F}: R \cup\{-\infty,+\infty\} \rightarrow[0,1]$ is called a distribution function if it is nondecreasing and left-continuous, $\mathrm{F}(0)=0$ and $\mathrm{F}(+\infty)=1$. The class of all probability distribution functions $\mathrm{F}$ is denoted by $\Lambda . D^{+}$is a subset of $\Lambda$ consisting of all functions $F \in \Lambda$ for which $l^{-} F(+\infty)=1$, where $l^{-} f(x)=\lim _{t \rightarrow x^{-}} f(t)$. The space $\Lambda^{+}$is 
partially ordered by the usual pointwise ordering of functions, that is, $F \leq G$ if and only if $F(t) \leq G(t)$ for all $t \in$ R. The maximal element for $\Lambda^{+}$with this order is the distribution function $\epsilon_{0}$ given by

$$
\epsilon_{0}(t)= \begin{cases}1, & \text { if } t>0 \\ 0, & \text { if } t \leq 0\end{cases}
$$

Definition 1.1. [14] A function $T:\{0,1] \times[0,1] \rightarrow[0,1]$ is a continuous triangular norm (briefly, a t-norm) if $T$ satisfies the following conditions:

(TN1) $T$ is commutative and associative;

(TN2) $T$ is continuous;

(TN3) $\mathrm{T}(\mathrm{a}, 1)=\mathrm{a}$ for all $a \in[0,1]$;

(TN4) $T(a, b) \leq T(c, d)$, whenever $a \leq c$ and $b \leq d$ for all $a, b, c, d \in[0,1]$.

Typical examples of continuous $t$-norms are $T_{M}(a, b)=\min (a, b), T_{P}(a, b)=a b$ and $T_{L}(a, b)=\max (a+b-$ $1,0)$ (the Lukasiewicz $\mathrm{t}$-norm). Recall [16] that if $\mathrm{T}$ is a $t$-norm and $\left\{x_{n}\right\}$ is a given sequence of numbers in $[0,1]$, then $T_{i=1}^{n} x_{i}$ is defined recurrently by

$$
T_{i=1}^{n} x_{i}=\left\{\begin{array}{cc}
x_{1}, & \text { if } n=1 \\
\mathrm{~T}\left(T_{i=1}^{n-1} x_{i}, x_{n}\right) & \text { if } n \geq 2
\end{array}\right.
$$

$T_{i=n}^{\infty} x_{i}$ is defined as $T_{i=1}^{\infty} x_{n+i}$.

Definition 1.2. [17] Let $X$ be a real linear space, $p \in R^{+}$with $0<p \leq 1$ and $T$ be a continuous t-norm. The triple $(X, \mu, T)$ is called a random $p$-normed space if a mapping $\mu: X \rightarrow D^{+}$(for any $x \in X, \mu(x)$ is denoted by $\mu_{x}$ ) satisfies the following conditions:

(RN1) $\mu_{x}(t)=\epsilon_{0}(t)$ for all $t>0$ if and only if $x=0$;

(RN2) $\mu_{\alpha x}(t)=\mu_{x}\left(\frac{t}{|\alpha|^{p}}\right)$ for all $x \in X$, all $t \geq 0$ and $\alpha \neq 0$;

(RN3) $\mu_{x+y}(t+s) \geq T\left(\mu_{x}(t), \mu_{y}(s)\right)$ for all $x, y \in X$, and all $t, s \geq 0$.

Note that every $\mathrm{p}$-normed space $(X,\|\cdot\|)$ defines a random $p$-normed space $\left(X, \mu, T_{M}\right)$, where $\mu_{x}(t)=\frac{t}{t+\|x\|}$ for all $t>0$ and $T_{M}$ is the minimum $t$-norm. This space is called the induced random $p$-normed space.

Definition 1.3. Let $(X, \mu, T)$ be a random p-normed space.

1. A sequence $\left\{x_{n}\right\}$ in $\mathrm{X}$ is said to be convergent to $\mathrm{x} \in \mathrm{X}$ if, for all $\mathrm{t}>0$ and $\lambda>0$, there exists a positive integer $\mathrm{N}$ such that $\mu_{x_{n}-x}(t)>1-\lambda$ whenever $\mathrm{n} \geq \mathrm{N}$.

2. A sequence $\left\{x_{n}\right\}$ in $\mathrm{X}$ is called Cauchy if, for all $\mathrm{t}>0$ and $\lambda>0$, there exists a positive integer $\mathrm{N}$ such that $\mu_{x_{n}-x_{m}}(t)>1-\lambda$ whenever $n \geq m \geq N$.

3. The random $p$-normed space $(X, \mu, T)$ is said to be complete if every Cauchy sequence in $X$ is convergent to a point in $X$.

Theorem 1.4. [14] If $(X, \mu, T)$ is a random normed space and $\left\{x_{n}\right\}$ is a sequence of $X$ such that $x_{n} \rightarrow x$, then $\lim _{n \rightarrow \infty} \mu_{x_{n}}(t)=\mu_{x}(t)$. 
Now, we consider the following functional equation:

$f(k x+y)+f(k x-y)=2 f(x+y)+2 f(x-y)+2\left(k^{2}-2\right) f(x)-2 f(y)$

for all $x, y \in X$. Then the functional equation (1.2) is equivalent to the standard Euler-Lagrange equation (1.1) and so, it is called a quadratic functional equation since the function $f(x)=x^{2}$ is its solution. Note that (1) if we put $x=y=0$, then $f(0)=0,(2) f\left(k^{n} x\right)=k^{2 n} f(x)$ for all $x \in X$ and $n \in Z^{+}$, and (3) $f(-x)=f(x)$ for all $x \in X$ in the functional equation (1.2). Motivated by the above facts, we investigate the generalized Hyers-Ulam stability problem of the functional equation (1.2) in random p-normed spaces (in the sense of Scherstnev) under the minimum $t$-norm $T_{M}$ using direct methods and fixed-point methods.

Throughout this paper, let $\mathrm{X}$ be a real linear space, $\left(\mathrm{X}, \mu^{\prime}, T_{M}\right)$ be a random $\mathrm{p}$-normed space and $\left(\mathrm{Y}, \mu, T_{M}\right)$ be a complete random $p$-normed space. For any mapping $\mathrm{f}: \mathrm{X} \rightarrow \mathrm{Y}$, we define

$$
\Delta f(x, y)=f(k x+y)+f(k x-y)-2 f(x+y)-2 f(x-y)-2\left(k^{2}-2\right) f(x)+2 f(x)
$$

for all $x, y \in X$ and any fixed positive integer $k \in Z^{+}$.

\section{Stability of the equation (1.2) in random p-normed spaces}

2.1. Directed Method. In this subsection, we prove the generalized Hyers-Ulam stability of the equation $\Delta \mathrm{f}(\mathrm{x}, \mathrm{y})=0$ in a random $\mathrm{p}$-normed space for $0<\mathrm{p} \leq 1$, by the standard Hyers' direct method.

Theorem 2.1. Let $\varphi: X^{2} \rightarrow Z$ be a function such that, for some $0<\alpha<k^{2}$,

$\mu_{\varphi(k x, k y)}^{\prime}(t) \geq \mu_{\alpha \varphi(x, y)}^{\prime}(t)$

and $\lim _{n \rightarrow \infty} \mu_{\varphi\left(k^{n} x, k^{n} y\right)}\left(k^{2 n p} t\right)=1$ for all $x, y \in X$ and all $t>0$. If $f: X \rightarrow Y$ is a mapping with

$f(0)=0$ such that

$\mu_{\Delta f(x, y)}(t) \geq \mu_{\varphi(x, y)}^{\prime}(t)$

for all $x, y \in X$ and all $t>0$, then there exists a unique quadratic mapping $Q: X \rightarrow Y$ such that

$\mu_{f(x)-Q(x)}(t) \geq \mu_{\varphi(x, 0)}^{\prime}\left(2^{p}\left(k^{2 p}-\alpha^{p}\right) t\right)$

for all $x \in X$ and all $t>0$.

Proof. Substituting $y=0$ in (2.2), we have

$\mu_{f(x)-\frac{f(k x)}{k^{2}}}(t) \geq \mu_{\varphi(x, 0)}^{\prime}\left(\left(2 k^{2}\right)^{p} t\right)$

for all $x \in X$ and all $t>0$. Replacing $\mathrm{x}$ by $k^{n} x$ in (2.4), we have

$$
\mu_{\frac{f\left(k^{n} x\right)}{k^{2 n}}-\frac{f\left(k^{n+1} x\right)}{k^{(2 n+1)}}}(t) \geq \mu_{\varphi\left(k^{n} x, 0\right)}^{\prime}\left(k^{2 n p} \cdot\left(2 k^{2}\right)^{p} t\right) \geq \mu_{\varphi(x, 0)}^{\prime}\left(\left(\frac{k^{2}}{\alpha}\right)^{n p} \cdot\left(2 k^{2}\right)^{p} t\right)
$$

for all $x \in X$ and all $t>0$. Since $f(x)-\frac{f\left(k^{n} x\right)}{k^{2 n}}=\sum_{j=0}^{n-1}\left(\frac{f\left(k^{j} x\right)}{k^{2 j}}-\frac{f\left(k^{j+1} x\right)}{k^{2(j+1)}}\right)$, 


$$
\begin{aligned}
& \mu_{f(x)-\frac{f\left(k^{n} x\right)}{k^{2 n}}}\left(\sum_{J=0}^{n-1} \frac{1}{\left(2 k^{2)^{p}}\right.}\left(\frac{\alpha}{k^{2}}\right)^{j p} t\right) \\
& =\mu_{\sum_{j=0}^{n-1}\left(\frac{f\left(k^{j} x\right)}{k^{2 j}}-\frac{f\left(k^{j+1} x\right)}{k^{2(j+1)}}\right)}\left(\sum_{J=0}^{n-1} \frac{1}{\left(2 k^{2}\right)^{p}}\left(\frac{\alpha}{k^{2}}\right)^{j p} t\right) \\
& \geq T_{M j=0}^{n-1} \frac{\mu_{f\left(k^{j} x\right)}}{k^{2 j}}-\frac{f\left(k^{j+1} x\right)}{k^{2(j+1)}}\left(\frac{1}{\left(2 k^{2}\right)^{p}}\left(\frac{\alpha}{k^{2}}\right)^{j p} t\right) \\
& =T_{M}\left(\mu_{\varphi(x, 0)}^{\prime}(t), \cdots, \mu_{\varphi(x, 0)}^{\prime}(t)\right) \\
& =\mu_{\varphi(x, 0)}^{\prime}(t)
\end{aligned}
$$

for all $x \in X$ and all $t>0$. Replacing $x$ by $k^{m} x$ in (2.5), we obtain

$\frac{\mu_{f\left(k^{\left.m_{x}\right)}\right.}}{k^{2 m}}-\frac{f\left(k^{\left.n+m_{x}\right)}\right.}{k^{2(n+m)}}(t) \geq \mu_{\varphi(x, 0)}^{\prime}\left(\frac{\left(2 k^{2}\right)^{p}}{\sum_{j=m}^{n+m-1}\left(\frac{\alpha}{k^{2}}\right)^{j p}} t\right)$

for all $x \in X$ and all $m, n \in Z$ with $n>m \geq 0$. It follows from

$$
\lim _{n, m \rightarrow \infty} \mu_{\varphi(x, 0)}^{\prime}\left(\frac{\left(2 k^{2}\right)^{p}}{\sum_{j=m}^{n+m-1}\left(\frac{\alpha}{k^{2}}\right)^{j p}} t\right)=1
$$

that the sequence $\left\{\frac{f\left(k^{n} x\right)}{k^{2 n}}\right\}$ is Cauchy in $\left(Y, \mu, T_{M}\right)$, and so it converges to some point $\mathrm{Q}(\mathrm{x}) \in \mathrm{Y}$. We can define a mapping $\mathrm{Q}: \mathrm{X} \rightarrow \mathrm{Y}$ by $\mathrm{Q}(\mathrm{x})=\lim _{n \rightarrow \infty} \frac{f\left(k^{n} x\right)}{k^{2 n}}$ for all $x \in X$ and all $t>0$. Fix $x \in X$ and put $m=0$ in (2.6). Then we obtain

$\mu_{f(x)-\frac{f\left(k^{n} x\right)}{k^{2 n}}}(t) \geq \mu_{\varphi(x, 0)}^{\prime}\left(\frac{\left(2 k^{2}\right)^{p}}{\sum_{j=0}^{n-1}\left(\frac{\alpha}{k^{2}}\right)^{j p}} t\right)$

for all $x \in X$ and all $t>0$. For every $\mathrm{s}>0$,

$$
\begin{aligned}
& \mu_{f(x)-Q(x)}(s+t) \\
& \geq T_{M}\left(\mu_{f(x)-\frac{f\left(k^{n} x\right)}{k^{2 n}}}(t), \mu_{\frac{f\left(k^{n} x\right)}{k^{2 n}}-Q(x)}(s)\right) \\
& \geq T_{M}\left(\mu_{\varphi(x, 0)}^{\prime}\left(\frac{\left(2 k^{2}\right)^{p}}{\sum_{j=0}^{n-1}\left(\frac{\alpha}{k^{2}}\right)^{j p}} t\right), \mu_{\frac{f\left(k^{n} x\right)}{k^{2 n}}-Q(x)}(s)\right)
\end{aligned}
$$

for all $x \in X$ and all $t>0$. Taking the limit as $\mathrm{n} \rightarrow \infty$ in (2.7), we obtain

$\mu_{f(x)-Q(x)}(s+t) \geq \mu_{\varphi(x, 0)}^{\prime}\left(2^{p}\left(k^{2 p}-\alpha^{p}\right) t\right)$.

Thus, since $s$ is arbitrary and by passings $\rightarrow 0$ in (2.8), we have 


$$
\mu_{f(x)-Q(x)}(t) \geq \mu_{\varphi(x, 0)}^{\prime}\left(2^{p}\left(k^{2 p}-\alpha^{p}\right) t\right)
$$

for all $x \in X$ and $t>0$. Thus, the condition (2.3) holds for all $x \in X$ and all $t>0$.

If we replace $(\mathrm{x}, \mathrm{y})$ by $\left(k^{n} x, k^{n} y\right)$ in (2.2), then

$\frac{\mu_{\Delta f\left(k^{n} x, k^{n} y\right)}}{k^{2 n}}(t) \geq \mu_{\varphi\left(k^{2} x, k^{2} y\right)}^{\prime}\left(k^{2 n p} t\right)$

for all $x, y \in X$ and all $t>0$. Letting $\mathrm{n} \rightarrow \infty$ in (2.9), we find that $\mu_{\Delta Q(x, y)}=1$ for all $t>0$, which implies $\Delta Q(x, y)=0$ for all $x, y \in X$. Therefore, the mapping $Q$ is quadratic.

To prove the uniqueness of the quadratic mapping $Q$, let us assume that there exists another mapping $\widetilde{Q}: X \rightarrow$ $Y$ which satisfies (2.3). For fixed $x \in X, \mathrm{Q}\left(k^{n} x\right)=k^{2 n} Q(x)$ and $\widetilde{Q}\left(k^{n} x\right)=k^{2 n} \tilde{Q}(x)$ for all $\mathrm{n} \in Z^{+}$. It follows from (2.3) that

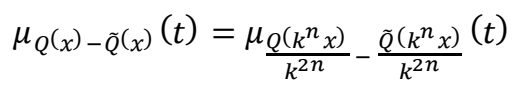

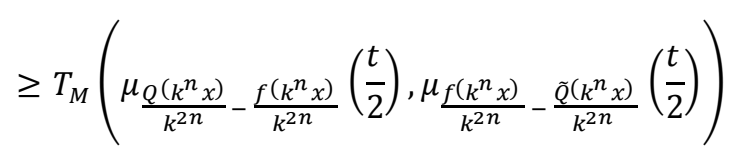

$$
\begin{aligned}
& \geq \mu_{\varphi(x, 0)}^{\prime}\left(\frac{2^{p}\left(k^{2 p}-\alpha^{p}\right)}{2}\left(\frac{k^{2}}{\alpha}\right)^{n p} t\right) .
\end{aligned}
$$

Since $\lim _{n \rightarrow \infty}\left(\frac{2^{p}\left(k^{2 p}-\alpha^{p}\right)}{2} \cdot \frac{k^{2 n p}}{\alpha^{n p}} t\right)=\infty$, we have $\mu_{Q(x)-\widetilde{Q}(x)}(t)=1$ for all $t>0$. Thus $Q(x)=\tilde{Q}(x)$

For all $x \in X$. This completes the proof.

Theorem 2.2. Let $\varphi: X^{2} \rightarrow Z$ be a function such that, for some $k^{2}<\alpha$,

$\mu_{\varphi\left(\frac{x}{k}, \frac{y}{k}\right)}^{\prime}(t) \geq \mu_{\varphi(x, y)}^{\prime}\left(\alpha^{p} t\right)$

and $\lim _{n \rightarrow \infty} \mu_{k^{2 n} \varphi\left(\frac{x}{k^{n}}, \frac{y}{k^{n}}\right)}^{\prime}(t)=1$ for all $x, y \in X$ and all $t>0$. If $f: X \rightarrow Y$ is a mapping with $f(0)=0$ which satisfies (2.2), then there exists a unique quadratic mapping $Q: X \rightarrow Y$ such that

$\mu_{f(x)-Q(x)}(t) \geq \mu_{\varphi(x, 0)}^{\prime}\left(2^{p}\left(\alpha^{p}-k^{2 p}\right) t\right)$.

for all $x \in X$ and all $t>0$.

Proof. Substituting $y=0$ in (2.2), we have

$\mu_{f(x)-k^{2} f\left(\frac{x}{k}\right)}(t) \geq \mu_{\varphi(x, 0)}^{\prime}\left((2 \alpha)^{p} t\right)$

for all $x \in X$ and all $t>0$. Replacing $x$ by $\frac{x}{k^{n}}$ in (2.12), we have

$$
\mu_{k^{2 n} f\left(\frac{x}{k^{n}}\right)-k^{2(n+1)} f\left(\frac{x}{k^{n+1}}\right)}(t) \geq \mu_{\varphi(x, 0)}^{\prime}\left((2 \alpha)^{p}\left(\frac{\alpha}{k^{2}}\right)^{n p} t\right)
$$

for all $x \in X$ and all $t>0$. Since $f(x)-k^{2 n} f\left(\frac{x}{k^{n}}\right)=\sum_{j=0}^{n-1}\left(k^{2 j} f\left(\frac{x}{k^{j}}\right)-k^{2(j+1)} f\left(\frac{x}{k^{j+1}}\right)\right)$, 
$\mu_{f(x)-k^{2 n} f\left(\frac{x}{k^{n}}\right)}\left(\frac{\sum_{j=0}^{n-1}\left(\frac{k^{2}}{\alpha}\right)^{j p}}{(2 \alpha)^{p}} t\right) \geq \mu_{\varphi(x, 0)}^{\prime}(t)$

for all $x \in X$ and all $t>0$. Replacing $x$ by $\frac{x}{k^{m}}$ in (2.13), we obtain

$$
\mu_{k^{2 m} f\left(\frac{x}{k^{m}}\right)-k^{2(n+m)} f\left(\frac{x}{k^{n+m}}\right)}(t) \geq \mu_{\varphi(x, 0)}^{\prime}\left(\frac{(2 \alpha)^{p}}{\sum_{j=m}^{n+m-1}\left(\frac{k^{2}}{\alpha}\right)^{j p}} t\right)
$$

for all $x \in X$ and $n, m \in Z$ with $\mathrm{n}>\mathrm{m} \geq 0$. Then the sequence $\left\{k^{2 n} f\left(\frac{x}{k^{n}}\right)\right\}$ is Cauchy in $\left(Y, \mu, T_{M}\right)$, and so it converges to some point $\mathrm{Q}(\mathrm{x}) \in \mathrm{Y}$. Now we can define a mapping $Q: X \rightarrow Y$ by $Q(x)=\lim _{n \rightarrow \infty} k^{2 n} f\left(\frac{x}{k^{n}}\right)$ for all $x \in X$ and all $t>0$. The remaining assertion goes through in a similar method to the corresponding part of Theorem 2.1. This completes the proof.

Corollary 2.3. Let $X$ be a real linear space, $\left(Z, \mu^{\prime}, T_{M}\right)$ be a random $p$-normed space and $\left(Y, \mu, T_{M}\right)$ be a complete random $p$-normed space. Assume $\delta$ is a positive real number and $z_{0}$ in $Z$. If $f: X \rightarrow Y$ is a mapping with $f(0)=0$ which satisfies

$\mu_{\Delta f(x, y)}(t) \geq \mu_{\delta z_{0}}^{\prime}(t)$

for all $x \in X$ and all $t>0$, then there exists a unique quadratic mapping $Q: X \rightarrow Y$ such that

$\mu_{f(x)-Q(x)}(t) \geq \mu_{\delta z_{0}}^{\prime}\left(2^{p}\left(k^{2 p}-1\right) t\right)$

for all $x \in X$ and all $t>0$.

Proof. Let $\varphi: X^{2} \rightarrow Z$ be defined by $\varphi(x, y)=\delta z_{0}$. Then the proof follows from Theorem 2.1 by $\alpha=1$. This completes the proof.

Corollary 2.4. Let $X$ be a real linear space, $\left(Z, \mu^{\prime}, T_{M}\right)$ be a random $p$-normed space and $\left(Y, \mu, T_{M}\right)$ be $a$ complete random $p$-normed space. Assume $r$ is a positive real number with $r \neq 3$ and $z_{0} \in Z$. If $f: X \rightarrow Y$ is a mapping with $f(0)=0$ which satisfies

$\mu_{\Delta f(x, y)}(t) \geq \mu_{\left(\|x\|^{r}+\|y\|^{r}\right)_{z_{0}}}^{\prime}(t)$

for all $x, y \in X$ and all $t>0$, then there exists a unique quadratic mapping $Q: X \rightarrow Y$ such that

$\mu_{f(x)-Q(x)}(t) \geq \mu^{\prime}{\|x\|{ }^{r} z_{0}}\left(2^{p}\left|k^{2 p}-k^{p r}\right| t\right)$.

for all $x \in X$ and all $t>0$.

Proof. Let $\varphi: X^{2} \rightarrow Z$ be defined by $\varphi(x, y)=\left(\|x\|^{r}+\|y\|^{r}\right) z_{0}$. Then, the proof follows from Theorem 2.1 and Theorem 2.2 by $\alpha=k^{r}$. This completes the proof.

\subsection{Fixed Point Method.}

In this subsection, using the fixed-point method, we give the generalized Hyers-Ulam stability of the equation $\Delta f(x, y)=0$ in random $p$-normed spaces. Let us recall that a mapping $\mathrm{d}: X^{2} \rightarrow[0, \infty)$ is called a metric on a nonempty set $X$ if (i) $d(x, y)=0$ if and only if $\mathrm{x}=\mathrm{y}$, (ii) $d(x, y)=d(y, x)$, (iii) $d(x, z) \leq d(x, y)+d(y, z)$ for all $x, y, z \in X$. Before proceeding to the main results in this subsection, we give the fixed point theorem which plays an important role in proving our theorem: 
Theorem 2.5. [18] Suppose that $(\Omega, d)$ is a complete metric space and $J: \Omega \rightarrow \Omega$ is a strictly contractive mapping with Lipschitz constant $L<1$. Then, for each $x \in \Omega$, either $d\left(J^{n} x, J^{n+1} x\right)=\infty$ for all nonnegative integers $n \geq$ 0 or there exists a natural number $n_{0}$ such that

(1) $d\left(J^{n} x, J^{n+1} x\right)<\infty$ for all $n \geq n_{0}$ ；

(2) the sequence $\left\{J^{n} x\right\}$ is convergent to a fixed point $y^{*}$ of $J$;

(3) $y^{*}$ is the unique fixed point of $j$ in the set $\Lambda=\left\{y \in \Omega: d\left(J^{n_{0}} x, y\right)<\infty\right\}$;

(4) $d\left(y, y^{*}\right) \leq \frac{1}{1-L} d(y, J y)$ for all $y \in \Lambda$.

Theorem 2.6. Let $\varphi: X^{2} \rightarrow D^{+}\left(\varphi(x, y)\right.$ is denoted by $\left.\varphi_{x, y}\right)$ be a function such that, for some $0<\alpha<k^{2}$, $\varphi_{k x, 0}\left(\alpha^{p} t\right) \geq \varphi_{x, 0}(t)$

for all $x \in X$ and all $t>0$. If $f: X \rightarrow Y$ is a mapping with $f(0)=0$ such that

$\mu_{\Delta f(x, y)}(t) \geq \varphi_{x, y}(t)$

for all $x, y \in X$ and all $t>0$, then there exists a unique quadratic mapping $Q: X \rightarrow Y$ such that

$\mu_{f(x)-Q(x)}(t) \geq \varphi_{x .0}\left(2^{p}\left(k^{2 p}-\alpha^{p}\right) t\right)$

for all $x \in X$ and all $t>0$.

Proof. Putting $y=0$ in (2.20), we get

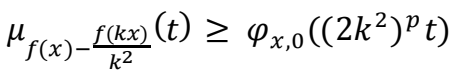

for all $x \in X$ and all $t>0$. Let $\Omega$ be a set of all mappings from $\mathrm{X}$ into $\mathrm{Y}$ and introduce a generalized metric on $\Omega$ as follows:

$$
d(g, h)=\inf \left\{c \in[0, \infty): \mu_{g(x)-h(x)}(c t) \geq \varphi_{x, 0}(t) \text { for all } x \in X\right\}
$$

where, as usual, $\inf \emptyset=+\infty$. It is easy to show that $(\Omega, d)$ is a complete metric space ([12]). Now, let us consider the mapping $J: \Omega \rightarrow \Omega$ defined by

$$
J_{g(x)}=\frac{1}{k^{2}} g(k x)
$$

for all $g \in \Omega$ and $x \in X$. Let $g, h$ in $\Omega$ and $c \in[0, \infty)$ be an arbitrary constant with $d(g, h)<c$. Then we have

$$
\mu_{g(x)-f(x)}(c t) \geq \varphi_{x, 0}(t)
$$

for all $x \in X$ and all $t>0$, whence

$\mu_{J g(x)-J h(x)}\left(\left(\frac{\alpha}{k^{2}}\right)^{p} c t\right) \geq \mu_{g(k x)-h(k x)}\left(\alpha^{p} c t\right) \geq \varphi_{k x, 0}\left(\alpha^{p} t\right) \geq \varphi_{x, 0}(t)$

for all $x \in X$ and all $t>0$, and so if $d(g, h)<c$, then 


$$
d(J g, J h) \leq \frac{\alpha^{p}}{k^{2 p}} d(g, h)
$$

for all $g, h \in \Omega$. Then, $\mathrm{J}$ is a strictly contractive self-mapping on $\Omega$ with the Lipschitz constant $L=\frac{\alpha^{p}}{k^{2 p}}<1$. Also, it follows from (2.20) that

$\mu_{f(x)-\frac{f(k x)}{k^{2}}}\left(\frac{t}{\left(2 k^{2}\right) p}\right) \geq \mu_{f(x)-J f(x)}\left(\frac{t}{\left(2 k^{2}\right) p}\right) \geq \varphi_{x, 0}(t)$

for all $x \in X$ and all $t>0$, which implies that

$d(f, J f) \leq \frac{1}{\left(2 k^{2}\right)^{p}}$.

Using Theorem 2.5, there exists a mapping $Q: X \rightarrow Y$, which is a unique fixed point of $\mathrm{J}$ in the set $\Omega_{1}=\{g \in \Omega: d(f, g)<\infty\}$ such that

$$
\mathrm{Q}(\mathrm{x})=\lim _{n \rightarrow \infty} \frac{f\left(k^{n} x\right)}{k^{2 n}}
$$

for all $\mathrm{x} \in \mathrm{X}$ since $\lim _{n \rightarrow \infty} d\left(J^{n} f, Q\right)=0$. Again, it follows from Theorem 2.5 that

$$
d(f, Q) \leq \frac{1}{1-L} d(f, J f) \leq \frac{1}{\left(2 k^{2}\right)^{p}(1-L)}=\frac{1}{2^{p}\left(k^{2 p}-\alpha^{p}\right)},
$$

which implies

$$
\mu_{f(x)-Q(x)}(t) \geq \varphi_{x .0}\left(2^{p}\left(k^{2 p}-\alpha^{p}\right) t\right)
$$

for all $x \in X$ and all $t>0$. Replacing $\mathrm{x}$ and $\mathrm{y}$ by $k^{n} x$ and $k^{n} y$ in (2.20), respectively,

$$
\mu_{\Delta Q(x, y)}(t)=\lim _{n \rightarrow \infty} \mu_{\Delta f\left(k^{n} x, k^{n} y\right)}\left(k^{2 n p} t\right) \geq \lim _{n \rightarrow \infty} \varphi_{k^{n} x, k^{n} y}\left(k^{2 n p} t\right)
$$

for all $x, y \in X$ and all $t>0$. It follows from $\lim _{n \rightarrow \infty} \varphi_{x, y}\left(\left(\frac{k^{2}}{\alpha}\right)^{n p} t\right)=1$ that $\Delta \mathrm{Q}(\mathrm{x}, \mathrm{y})=0$. Hence, the mapping $\mathrm{Q}$ is quadratic.

To prove the uniqueness, let us assume that there exists a quadratic mapping $Q^{\prime}: X \rightarrow Y$ which satisfies (2.21). Then $\mathrm{Q}^{\prime}$ is a fixed point of $\mathrm{J}$ in $\Omega_{1}$. However, it follows from Theorem 2.5 that $\mathrm{J}$ has only one fixed point in $\Omega_{1}$. Hence, we deduce that $Q=Q^{\prime}$. This completes the proof.

Theorem 2.7. Let $\varphi: X^{2} \rightarrow D^{+}$be a function such that, for some $k^{2}<\alpha$,

$\varphi_{x, 0}(t) \geq \varphi_{k x, 0}\left(\alpha^{p} t\right)$

for all $x \in X$ and all $t>0$. If $f: X \rightarrow Y$ is a mapping with $f(0)=0$ which satisfies (2.20), then there exists a unique quadratic mapping $Q: X \rightarrow Y$ such that

$\mu_{f(x)-Q(x)}(t) \geq \varphi_{x, 0}\left(2^{p}\left(\alpha^{p}-k^{2 p}\right) t\right)$

for all $x \in X$ and all $t>0$.

Proof. Let $\Omega$ and $\mathrm{d}$ be as in the proof of Theorem 2.2. Then $(\Omega, d)$ becomes a complete metric space and the mapping $J: \Omega \rightarrow \Omega$ defined by 


$$
J_{g(x)}=k^{2} g\left(\frac{x}{k}\right)
$$

for all $g \in \Omega, x \in X$. Then,

$$
d(J g, J h) \leq \frac{k^{2 p}}{\alpha^{p}} d(g, h)
$$

for all $g, h \in \Omega$. Then, $J$ is a strictly contractive self-mapping on $\Omega$ with the Lipschitz constant

$L=\frac{k^{2 p}}{\alpha^{p}}<1$. It follows from $(2.20)$ that $d(f, J f) \leq \frac{1}{(k \alpha)^{p}}$. Thus, we obtain

$$
d(f, Q) \leq \frac{1}{1-L} d(f, J f) \leq \frac{1}{2^{p}\left(\alpha^{p}-k^{2 p}\right)},
$$

which implies the inequality (2.20) holds for all $x \in X$ and all $t>0$. The remaining assertion goes through in a similar method to the corresponding part of Theorem 2.6. This completes the proof.

Corollary 2.8. Let $X$ be a real $p$-Banach spaces, and define $\mu_{x}(t)=\frac{t}{t+\|x\|}$ for all $x \in X$ and all $t>0$. Then $\left(X, \mu, T_{M}\right)$ is a complete random $p$-normed space. Define

$$
\varphi_{x, y}(t)=\frac{t}{t+\left(\|x\|^{r}+\|y\|^{r}\right)}
$$

for all $x, y \in X$ and all $t>0$ in which $0<r<3$. Assume that $f: X \rightarrow Y$ is a mapping with $f(0)=0$, which satisfies (2.20). Then, there exists a unique quadratic mapping $Q: X \rightarrow Y$ such that

$\mu_{f(x)-Q(x)}(t) \geq \frac{2^{p}\left(k^{2 p}-k^{p r}\right) t}{2^{p}\left(k^{2 p}-k^{p r}\right) t+\|x\|^{r}}$

for all $x, y \in X$ and all $t>0$, where $\alpha=k^{p}$. Hence, we have

$\|f(x)-Q(x)\| \leq \frac{\|x\|^{r}}{2^{p}\left(k^{2 p}-k^{p r}\right)}$

for all $x \in X$.

\section{Reference}

1. S.M. Ulam, Problems in Modern Mathematics, Science Editions, John Wiley \\& Sons, New York, USA, 1940.

2. D.H. Hyers, On the stability of the linear functional equation, Proc. Nat. Acad. Sci. USA 27 (1941), 222-224.

3. T. Aoki, On the stability of the linear transformation in Banach spaces, J. Math. Soc. Japan. 2 (1950), 64-66.

4. Th.M. Rassias, On the stability of the linear mapping in Banach spaces, Proc. Amer. Math. Soc. 72 (1978), 297-300.

5. P. Gãvruta, A generalization of the Hyers-Ulam-Rassias stability of approximately additive mappings, J. Math. Anal. Appl. 184 (1994), 431-436.

6. L. Cădariu, V. Radu, Fixedoints, and the stability of Jensen's functional equation, J. Iraq. Pure Appl. Math. 4 (2003), No. 1, Art. 4.

7. P.W. Cholewa, Remarks on the stability of functional equations, Aeq. Math. 27(1984), 76-86. 
8. P. Czerwik, On the stability of the quadratic mapping in normed spaces, Abh. Math. Sem. Univ. Hamburg, 62(1992), 59-64.

9. F. Skof, Proprieta locali e approssimazione di operatori, Rend. Sem. Mat. Fis. Milano, 53(1983), 113-129.

10. Y.J. Cho, TM. Rassias, R. Saadati, Stability of Functional Equations in Random Normed Spaces, Springer Optimization and Its Application 86, Springer New York, 2013.

11. , . Khodaei, ME. Gordji, S.S. Kim and Y.J. Cho, Approximation of radical functional equations related to quadratic and quartic mappings, J. Math. Anal. Appl. 395(2012), 284-297.

12. D. Mihet, V. Radu, On the stability of the additive Cauchy functional equation in random normed spaces, J. Math. Anal. Appl. 343(2008), 567-572.

13. J.M. Rassias, R. Saadati, G. Sadeghi, J. Vahidi, On nonlinear stability in various random normed spaces, J. Ineq. Appl. 2011, 2011:62.

14. B. Schweizer, A. Skar, Probability Metric Spaces, North-Holland Series in Probability and Applied Math. New York, USA 1983.

15. A.N. Sherstnev, On the notion of s random normed spaces, Dpkl. Akad. Nauk SSSR 149, $280-283$ (in Russian).

16. O. Hadz̃ić, E. Pap, M. Budincevi '\{c\}, Countable extension of triangular norms and their applications to the fixed-point theory in probabilistic metric spaces, Kybernetika 38(2002), 363-381.

17. Golet, Random \$p\$-normed spaces and applications to random functions, Istanbul Univ. Fen Fak. Mat. Fiz. Astro. Derg. 1(2004-2005), 31-42.

18. J.B. Dias, B. Margolis, A fixed point theorem of the alternative for contractions on a generalized complete metric space, Bull. Amer. Math. Soc. 74 (1968), 305-309. 